\title{
Agil arbeiten, lernen, Unternehmenswerte pflegen
}

\author{
Gabriele Korge, Agnes Bauer und Mara Sharma
}

\section{Steckbrief}

\begin{tabular}{l|l}
\hline Unternehmen & $\begin{array}{l}\text { eXXcellent solutions GmbH, Ulm } \\
\text { Liefert als IT-Dienstleister passgenaue Software-Lösungen für Unternehmen. } \\
\text { Ca. 200 Mitarbeitende an 5 Standorten in Deutschland }\end{array}$ \\
\hline Auftrag & $\begin{array}{l}\text { Die Lernenden sollten sich mit den Unternehmenswerten auseinandersetzen und } \\
\text { Wege finden, sie zu leben. }\end{array}$ \\
\hline Rollen & $\begin{array}{l}\text { Lernende: 7 Mitarbeitende an 3 Standorten, nach Unternehmenszugehörigkeit } \\
\text { und Aufgabenbereich durchmischt } \\
\text { Begleitende: ein Scrum Master für die methodische Begleitung, eine } \\
\text { Personalreferentin für die fachliche Begleitung } \\
\text { Auftraggebende: Geschäftsfühung, Resort Personal }\end{array}$ \\
\hline Ablauf & $\begin{array}{l}\text { In gut 2 Monaten wurden 3 Etappen von je 3 Wochen Dauer durchlaufen. Zum } \\
\text { Auftakt, zwischen den Etappen und zum Abschluss fanden Präsenzmeetings } \\
\text { statt. Der Lernaufwand entsprach ca. 1 Stunde pro Woche. }\end{array}$ \\
\hline
\end{tabular}

G. Korge $(\triangle)$

Fraunhofer-Institut für Arbeitswirtschaft und Organisation IAO, Stuttgart, Deutschland

E-Mail: gabriele.korge@iao.fraunhofer.de

A. Bauer

ZNL TransferZentrum für Neurowissenschaften und Lernen der Universität Ulm, Ulm, Deutschland

E-Mail: agnesbauer@web.de

M. Sharma

eXXcellent solutions GmbH, Human Resources, Stuttgart, Deutschland

E-Mail: mara.sharma@exxcellent.de

J. Longmuß et al. (Hrsg.), Agiles Lernen im Unternehmen,

https://doi.org/10.1007/978-3-662-62013-7_6 


\begin{tabular}{l|l}
\hline Technik & $\begin{array}{l}\text { Da zur Organisation der täglichen Projektarbeit JIRA genutzt wird, kam dieses } \\
\text { auch zur Unterstützung des Lernens zum Einsatz; zudem die Instant-Messaging- } \\
\text { Dienste Mattermost und Slack, Video- und Webkonferenzen sowie Server zur } \\
\text { Dokumentenablage. }\end{array}$ \\
\hline Besonderheiten & $\begin{array}{l}\text { • Agiles Lernen für Mitarbeitende, die bereits agil arbeiten } \\
\text { • Ein Lerngegenstand, Werte, mit dem man sich nur indirekt befassen kann und } \\
\text { der Emotionen anspricht. }\end{array}$ \\
\hline
\end{tabular}

\subsection{Anlass und Ziel}

Das Unternehmen eXXcellent solutions ist in den letzten Jahren stark gewachsen, insbesondere kamen neue Standorte hinzu. Um die Identität und das Zusammengehörigkeitsgefühl zu stärken, setzte die Geschäftsführung einen mehrstufigen Prozess zur Entwicklung und Verankerung von Unternehmenswerten auf, in den die Mitarbeiter aller Standorte aktiv eingebunden waren. Als ein erstes Ergebnis entstand ein Wertekanon, abgebildet über die drei Identitäts-Dreiecke „Wofür wir stehen“, „Wie wir miteinander umgehen“ und ,Wie wir die Dinge angehen“.

Als nächster Schritt vor der weiteren Implementierung sollte dieser Wertekanon nun auf Akzeptanz, Relevanz und Handhabbarkeit geprüft werden: War es gelungen, Werte abzubilden, die tatsächlich von Bedeutung für den Arbeitsalltag waren? Und konnte der Kanon Wegweiser sein für ein wertekonformes Verhalten? Um diesen Fragen nachzugehen, entschloss sich die Geschäftsführung, den Mitarbeitenden die Chance zur Auseinandersetzung mit den Werte-Dreiecken zu geben.

Die Personalreferentin entschied, dazu ein agiles Lernprojekt aufzusetzen: Diese Lernform wurde gewählt, weil sie den Lernprozess flexibel und offen gestaltet. Zudem entspricht sie der Arbeitsweise bei eXXcellent solutions und lässt ein arbeitsplatznahes Lernen zu, was ein Ausrollen, also ein Werte-Lernen vieler, ermöglicht.

\subsection{Vorbereitung: Ein besonderes Lernkonzept für ein besonderes Lernziel}

Zwei Softwareentwickler von eXXcellent solutions, die selbst agil arbeiten und dabei bedarfsorientiert die Rolle des Scrum Master oder des Product Owner einnehmen, waren auch für die methodische und fachliche Begleitung des agilen Lernens qualifiziert und hatten wiederholt Studierende im agilen Lernen begleitet. Allerdings war der Lerngegenstand Vorgehen in der Programmierung sowie JAVA als Programmiersprache - nicht vergleichbar mit der Aufgabe, sich mit Unternehmenswerten auseinanderzusetzen: Beim Lernen zu JAVA gab es durchaus ein „Richtig“ oder „Falsch“. In der Auseinandersetzung mit den Unternehmenswerten ging es hingegen um persönliches Erleben und subjektive Haltungen.

Ausgehend von diesen Überlegungen holte sich die Personalreferentin externe Unterstützung, um ein zum Lernziel passendes Konzept aufzusetzen. Eckpunkte waren: 
- eine rhythmische Lernorganisation mit gleichmäßigen Etappen von zwei bis maximal vier Wochen und einem Lernaufwand von ca. 1 Stunde je Woche für eine gute Planbarkeit und Vereinbarkeit mit der Arbeit,

- die Durchführung der Treffen zum Auftakt, Abschluss und zwischen den Etappen als Präsenzmeetings an wechselnden Standorten, um die auch persönliche Auseinandersetzung mit dem Thema zu erleichtern und die standortübergreifende Identität zu fördern,

- die Präsenz der Geschäftsführung zumindest zum Auftakt und zum Abschluss der Maßnahme, um die Bedeutung für eXXcellent solutions zu betonen und Gelegenheit zur direkten Kommunikation zu geben,

- ein Lernteam aus sechs bis acht Mitarbeitenden mit einer möglichst großen Bandbreite bezüglich Unternehmenszugehörigkeit, Standort und Arbeitsaufgaben - um unterschiedliche Sichtweisen einzufangen sowie neue Begegnungen zu ermöglichen und das Ausbilden einer gemeinsamen Identität zu fördern,

- die Freistellung der Lernenden von der Arbeit, um die Bedeutung des Projekts zu betonen und Mehrbelastung zu vermeiden,

- Lernaufgaben, die es den Lernenden ermöglichen, an eigenes Erleben anzuknüpfen wobei es ihnen selbst überlassen bleibt, mit welchen Werten sie sich befassen,

- die Bereitstellung alternativer Lernaufgaben, damit die Lernenden in der Planung eine Auswahl treffen und auch Aufgaben zurückweisen können,

- eine Ergebnisabnahme im Review, die nicht von „Richtig“ oder „Falsch“ geprägt ist, da es diese Art Fremdbewertung bei einem solchen Thema nicht geben kann.

\subsection{Umsetzung: Von der Idealvorstellung zum Machbaren}

Um es vorwegzunehmen: Nur ein Teil des Konzepts ließ sich in der gewünschten Weise umsetzen.

Die Lernorganisation entsprach in einigen Punkten dem Konzept: Es gab 3-Wochen-Etappen, in welchen die Lernenden je ca. 3 Stunden für das Lernen aufwendeten - alle Treffen waren Präsenzmeetings und dauerten ca. 1,5 Stunden (siehe Abb. 6.1). Allerdings fanden die Treffen nicht an wechselnden Orten statt, sondern ausnahmslos am Standort Ulm.

Ein wesentlicher Grund dafür war, dass fünf der Lernenden vom Standort Ulm kamen, während nur zwei weitere Standorte durch jeweils einen Mitarbeiter vertreten waren. Auch sonst war das Lernteam nicht so heterogen zusammengesetzt wie ursprünglich geplant: Das agile Lernprojekt sollte noch vor der Sommerpause abgeschlossen werden, und so wurden die Teilnehmenden nach Verfügbarkeit und Vereinbarkeit mit der Arbeit ausgewählt. Die Gruppe der Lernenden bestand aus

- zwei Softwareentwicklern mit langjähriger Unternehmenszugehörigkeit, 


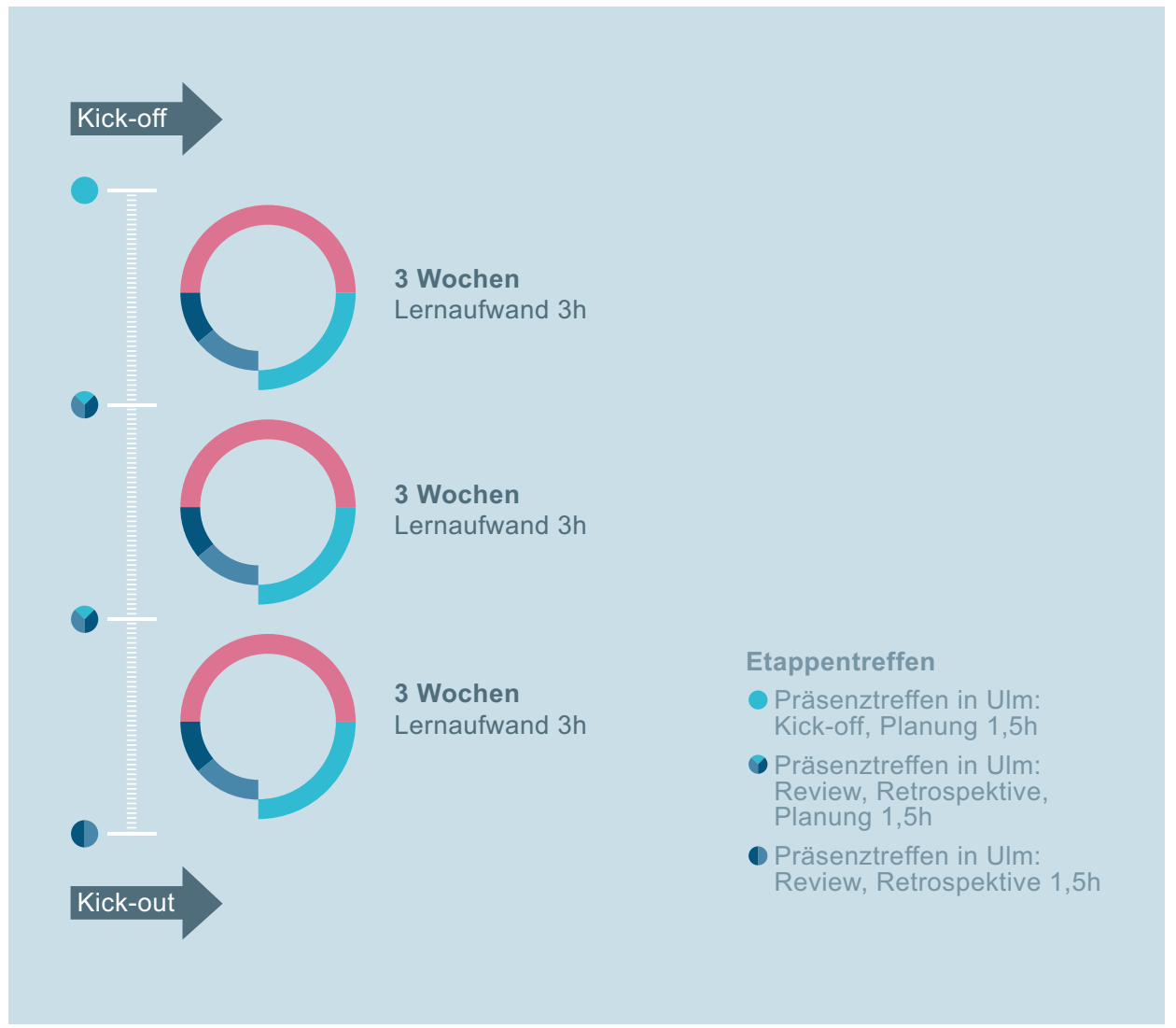

Abb. 6.1 Die Lernorganisation

- vier angehenden Softwareentwicklern in der studentischen Ausbildung mit nur kurzer Zugehörigkeit,

- einer Mitarbeiterin aus einem indirekten Bereich mit wenigen Monaten Unternehmenszugehörigkeit.

Der enge Zeitplan führte auch dazu, dass die Geschäftsführung nicht an den Treffen teilnehmen konnte. Sie wurde von der Personalreferentin vertreten, die auch die Rolle der fachlichen Begleitung übernahm. Für die methodische Begleitung erhielt sie Unterstützung von einem Kollegen, der das agile JAVA-Lernen bereits mehrfach begleitet hatte.

Um den Lernenden wie beabsichtigt die Wahl zu lassen, auf welche Weise sie die Themen bearbeiten wollen, und um gleichzeitig die umfassende Auseinandersetzung mit Werten sicherzustellen, wurden mehrere Aufgabenbündel mit je drei Aufgaben ausgearbeitet. In der Planung konnte sich das Team dann jeweils entscheiden, welches Aufgabenbündel es als nächstes bearbeiten wollte, und auch Aufgabenbündel zurückweisen. Tatsächlich wurde ein Aufgabenbündel abgelehnt und nicht bearbeitet. Abb. 6.2 zeigt die drei bearbei- 


\section{Die Unternehmenswerte von XX: Das habe ich erlebt!}

Welches eingängige Erlebnis zu gelebten oder verletzten Unternehmenswerten hattest du?

1. Schildere dein Erlebnis Kollegen und tausche dich aus: Welche Werte waren betroffen? Wie ging es dir?

2. Fasse ein Vorhaben: Was kannst du persönlich tun, um den betroffenen Wert (noch) besser zu leben? Setze dein Vorhaben um!

3. Setze dich mit deinen Erfahrungen zum Vorhaben auseinander und fasse ein Fazit. Arbeite eine Strategie aus, wie du deine Erfolge mit Kollegen teilen kannst.

\section{Wie virtuelle Zusammenarbeit besser gelingt!}

Häufig arbeiten wir nur virtuell mit Kollegen zusammen. Unsere Werte zu leben kann dann eine besondere Herausforderung darstellen.

1. Betrachtet typische Situationen: In welchen Situationen fällt es schwerer, die Werte zu leben? Um welche Werte geht es? Tragt eure Erlebnisse im Lernteam zusammen.

2. Bildet Kleingruppen und analysiert das Geschehen zu einer der Situationen. Leitet Maßnahmen ab, um die Werte besser leben zu können: Was kann technisch, organisatorisch oder am Verhalten Einzelner geändert werden? Teilt die Ergebnisse der Kleingruppen im gesamten Lernteam.

3. Setzt euer Vorhaben in die Tat um oder spielt die Situation in einem Rollenspiel durch. Tragt eure Eindrücke zusammen und zieht ein Fazit: Was empfehlt ihr Kollegen? Welche weiteren Maßnahmen oder welche Unterstützung von der Geschäftsführung braucht es?

\section{Was macht XX zu einem echten „Great Place to Work“?}

Stellt euch einen Typ vor, den ihr nicht mögt und der mit seinem Arbeitgeber angibt.

1. Mit welchem Wert wollt ihr dagegenhalten? Sammelt eure Angeber-Werte im Team und erstellt euer Werte-Ranking.

2. Erstellt eine Angeber-Story zu einem eurer Angeber-Werte. Ihr dürft dabei auch so richtig angebermäßig übertreiben!

3. Beschreibt die Lücke zwischen eurer Angeber-Story und dem Ist-Bild. Schlagt einen Kommunikationsweg vor, um das Defizit-Bild an die Geschäftsführung zu kommunizieren.

Abb. 6.2 Die Lernaufgaben zu den Unternehmenswerten in Kurzform

teten Aufgabenbündel in gekürzter Form und gibt einen Eindruck davon, wie sich die Lernenden in den Etappen mit den Unternehmenswerten auseinandersetzen sollten.

In den Etappen tauschten sich die Lernenden sowohl im großen Kreis als auch in wechselnden Kleingruppen aus. Zudem fanden Gespräche über das Lernteam hinaus mit Ar- 
beitskollegen oder spontan in der Kaffeeküche statt. Einmal entwickelte sich im Mehrpersonenbüro eine spontane Diskussion zu einem aktuellen Werte-Thema: Die konkrete Situation wurde analysiert, eine Maßnahme wurde entwickelt und in die Tat umgesetzt. Dabei näherten sich die Lernenden dem Thema auf ganz unterschiedliche Weise. Eine Kleingruppe spielte zur Erprobung eines Lösungsansatzes die Situation in einem Rollenspiel durch - eine Methode, die von der Mehrheit der Lernenden jedoch ausdrücklich abgelehnt wurde.

Zum standortübergreifenden Austausch nutzten die Lernenden IuK-Techniken aus dem Arbeitsalltag: das Projektmanagement-Tool JIRA, um Ergebnisse zum Lernen im gesamten Lernteam auszutauschen oder für das Review bereit zu stellen, Video- und WebKonferenzen zur live-online Kommunikation und Instant-Messaging-Dienste zum Chatten.

Zeitlicher Schwerpunkt der Etappentreffen war das Review. Hier schilderten die Lernenden erlebte Situationen, ihr Vorgehen zur Analyse, Lösungsansätze und erste Erfahrungen damit. Das Review glich folglich über weite Strecken eher einem erneuten Austausch - in der großen Runde und mit der fachlichen Begleiterin - als dass es den Charakter einer Ergebnisabnahme hatte.

Die Retrospektive fiel in aller Regel recht kurz aus. Der methodische Begleiter bediente sich sehr geschickt der Methoden und Ansätze aus dem Scrum Kontext, um die Aufmerksamkeit auf das Thema der Prozessbetrachtung zu lenken und den Diskussionsraum zu öffnen. Zum Beispiel forderte er die Lernenden anhand der 3-Wort-Methode dazu auf, die letzte Etappe anhand von drei Worten zu beschreiben. Durch Clusterung der Nennungen am Flipchart machte er auf einfache Weise das allgemeine Stimmungsbild sichtbar. Zudem sprach er organisatorische und methodische Aspekte sowie die Zugänglichkeit des Themas an: Wird Relevantes thematisiert? Werden die Aufgaben verstanden?

\subsection{Beobachtungen und Lessons Learned}

Letztlich zeigte sich, dass die vielen Abweichungen vom ursprünglichen Konzept geringere Auswirkungen hatten als befürchtet. Spürbar war jedoch, dass die Entlastung der Lernenden durch eine Freistellung von Arbeitsaufgaben nicht wirklich funktioniert hatte: Wenn dringende Arbeiten anstehen, kann man sich dem nicht entziehen! Hier würden verbindliche Absprachen zum Gesamtpensum gewährleisten, dass die Gesamtbelastung für einzelne Lernende nicht zu groß wird.

Dies sowie die eingeschränkte Freiwilligkeit der Teilnahme hat sich auf die Motivation der Lernenden nicht spürbar ausgewirkt: Die Lernenden bezogen ihre Motivation aus der Bearbeitung eigener Erlebnisse und der jeweiligen Betroffenheit. Diese persönlichen Erlebnisse waren dann auch jeweils Ausgangspunkt für einen regen Austausch über eine Vielzahl an Werten. Hier machte sich die eher geringe standort- oder aufgabenbezogene Heterogenität letztlich nicht negativ bemerkbar. 
Die zentralen Lessons Learned zum agilen Werte-Lernen stützen sich auf Äußerungen einer Mehrheit der Lernenden im Kick-out: Sie hatten das Gefühl, letztlich für sich selbst nichts gelernt zu haben, sondern vor allem Ergebnisse geliefert zu haben, die der Geschäftsführung und der Personalreferentin im Entwicklungsprozess um die Unternehmenswerte weiterhelfen würden. Gleichzeitig konnte jedoch aus der Beobachterperspektive festgestellt werden, dass sich alle Lernenden gute Gedanken zum Thema gemacht und Wege zur Annäherung an die Thematik erarbeitet hatten.

Erklärbar wird diese Diskrepanz bei näherer Betrachtung dessen, was als primäres Lernziel definiert, im Kick-off vermittelt, mit den Aufgaben verfolgt und im Review nachgehalten wurde: So, wie die Aufgaben aufgebaut waren, war das Lernziel dann erreicht, wenn ein Vorhaben gefasst und umgesetzt wurde - wenn zum Beispiel eine Angeber-Story verfasst und die Lücke zwischen der Ist- und der Soll-Situation beschrieben wurde (s. Abb. 6.2).

Aus Sicht eines Didaktikers dagegen haben die Lernenden durch die Bearbeitung der Aufgaben sehr wohl Lernerfolge vorzuweisen: Sie haben gelernt Situationen zu analysieren, geeignete Vorhaben zu fassen, diese in die Tat umzusetzen und das Ergebnis zu bewerten.

Genau dieses Vorgehen haben sie aber - so wie die Reviews und Retrospektiven durchgeführt wurden - nie reflektierend betrachtet. Sie haben sich nie gefragt: Wie gehe ich genau vor? Ist es gut so? Kann ich das reproduzieren? Und sie haben genau dazu nie ein Feedback erhalten. Dies ist nachvollziehbar, da diese Reflexionsübung so nicht in den Lernaufgaben formuliert war. Zudem war der methodische Begleiter als Scrum Master eher für Prozesse der Software-Entwicklung qualifiziert und hatte keine Schulung zur Begleitung von kommunikativen und sozialen Lernprozessen erhalten.

Verbesserungsmöglichkeiten zeigen sich somit in der Vermittlung des Lernziels, in der Durchführung von Review und Retrospektive sowie evtl. in der Formulierung von Lernaufgaben, die dieses bewusste Reflektieren - „Wie genau bin ich jetzt vorgegangen, um die Werte zu pflegen, und war das hilfreich?“ - in der Aufgabenstellung mit aufnehmen könnten.

\subsection{Fazit: Was bleibt}

Das primäre Ziel - die Lernenden erfahren, wie sie sich mit den Unternehmenswerten auseinandersetzen können - wurde also durchaus erreicht, wenn dies den Lernenden auch nicht immer so bewusst war.

Auch aus Unternehmenssicht hat sich das agile Lernen als gutes Instrument zur Unterstützung von Wertepflege und Identitätsförderung erwiesen. Die im Lernteam und darüber hinaus - in der Kaffeeküche, im Großraumbüro - angestoßenen Diskussionen haben sicher Spuren hinterlassen und eine Reihe von Mitarbeitenden und Führungskräften für das Thema sensibilisiert. Zur Lernaufgabe „Great Place to Work“ entwickelten die Lernenden einen Markting-Flyer (s. Abb. 6.3) und zwei Comics zur Identitätsförderung. In den drei Etappen ergriffen die Lernenden selbst Initiative und stießen eine Reihe von Verbesse- 


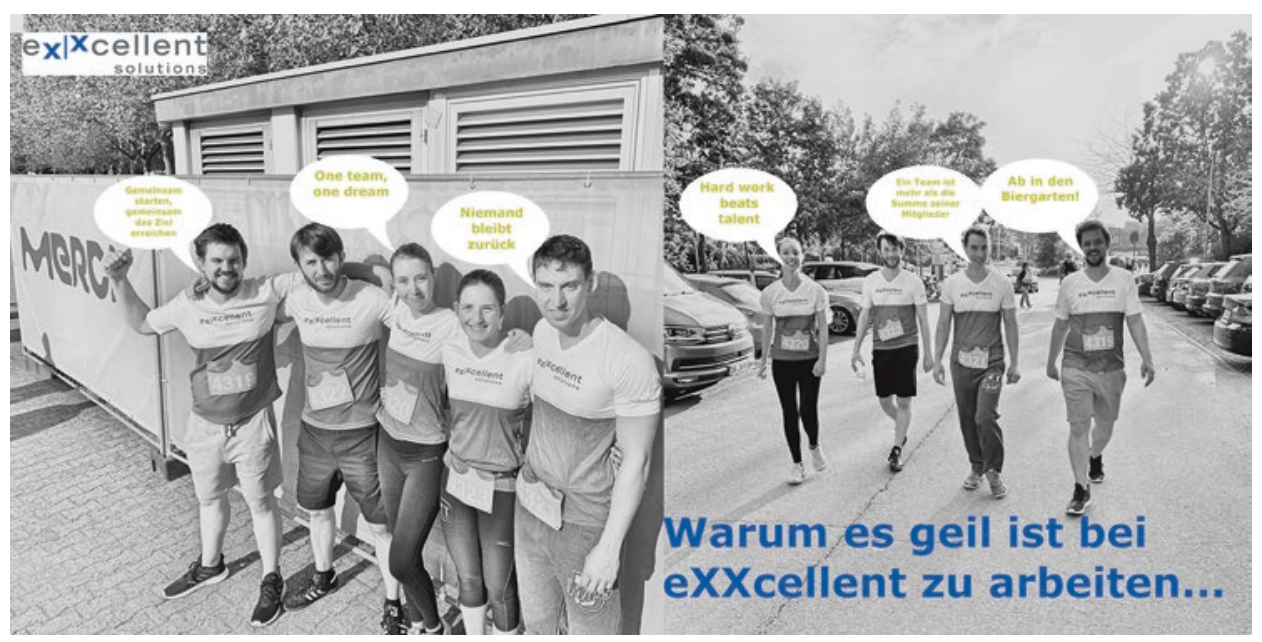

Abb. 6.3 Ergebnis zur Lernaufgabe „Great Place to Work“

rungsmaßnahmen an oder entwickelten Vorschläge, wie die Werte besser gelebt werden können. Beispiele sind Wege zum entspannten Arbeiten im Mehrpersonenbüro, die Idee für eine „Willkommensmappe“ für neue Kollegen oder ein Konzept für eine bessere technisch-organisatorische Unterstützung der bereichs- und standortübergreifenden Zusammenarbeit.

Einige Ideen der Lernenden konnten gut in laufende Maßnahmen integriert werden. Der Vorschlag einer Willkommensmappe wurde weiter entwickelt zu einer EmailKampagne mit Informationen für Bewerber, einem überarbeiteten Onboarding-Booklet und einem kleinen Willkommensgeschenk. Weiterhin wurde beschlossen, die erste Woche der neuen Kollegen ins Visier zu nehmen, um ihr Ankommen im Unternehmen besser zu gestalten und sie gut mit auf den Weg zu nehmen.

Auch die von Lernenden erprobten neuen Organisationsformen und technischen Lösungen zu gemeinsamem Arbeiten und Lernen über Standorte hinweg konnten in ein bereits angestoßenes Programm zur Unterstützung der virtuellen Zusammenarbeit einfließen. Alle Standorte wurden auf eine Software umgestellt, mit der Videokonferenzen und Screensharing möglich sind. Auch Lightning Talks mittels Videobrücke werden mittlerweile standortübergreifend durchgeführt.

Darüber hinaus berichten die Teilnehmer des agilen Lernens, dass die jeweiligen persönlichen Ziele, die sie sich im Lernen gesetzt hatten, weiterhin bedeutsam sind für ihr Arbeiten. 
Open Access Dieses Kapitel wird unter der Creative Commons Namensnennung - Weitergabe unter gleichen Bedingungen 4.0 International Lizenz (http://creativecommons.org/licenses/ by-sa/4.0/deed.de) veröffentlicht, welche die Nutzung, Vervielfältigung, Bearbeitung, Verbreitung und Wiedergabe in jeglichem Medium und Format erlaubt, sofern Sie den/die ursprünglichen Autor(en) und die Quelle ordnungsgemäß nennen, einen Link zur Creative Commons Lizenz beifügen und angeben, ob Änderungen vorgenommen wurden. Wenn Sie das Buch oder Teile daraus remixen, verändern oder anderweitig direkt darauf aufbauen, dürfen Sie Ihre Beiträge nur unter derselben Lizenz wie das Original verbreiten.

Die in diesem Kapitel enthaltenen Bilder und sonstiges Drittmaterial unterliegen ebenfalls der genannten Creative Commons Lizenz, sofern sich aus der Abbildungslegende nichts anderes ergibt. Sofern das betreffende Material nicht unter der genannten Creative Commons Lizenz steht und die betreffende Handlung nicht nach gesetzlichen Vorschriften erlaubt ist, ist für die oben aufgeführten Weiterverwendungen des Materials die Einwilligung des jeweiligen Rechteinhabers einzuholen.

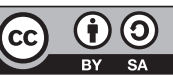

